\title{
WHY BUY THE ORIGINAL, IF THE FAKE CAN DO THE SAME? (CAN IT?) - THE DEMAND FOR COUNTERFEIT SPORTS GEAR IN AN EMERGING MARKET
}

\author{
Barbara Stöttinger, Vienna University of Economics \& Business, Austria \\ Elfriede Penz, Vienna University of Economics \& Business, Austria
}

\begin{abstract}
Counterfeiting has emerged as a major problem for global marketers. It has risen dramatically over the past years and now represents some $5-8 \%$ of total world trade (Balfour, 2005). While supply side deterrents may still be helpful in this context, the active role of the consumer as 'accomplice' (Bloch, Bush, \& Campbell, 1993) calls for demand side counteractions. As long as the demand is thriving, counterfeiters will always find new ways to serve customers (e.g., Albers-Miller, 1999). Substantial research has looked into what drives consumers to buy the fake rather than the original product and various influences were identified (Eisend \& Schuchert-Güler, 2006; Staake, Thiesse, \& Fleisch, 2009). Many of these studies have focused either on industrialized countries (e.g.,Gistri, Romani, Pace, Gabrielli, \& Grappi, 2009; Penz \& Stöttinger, 2005)or emerging markets in SE-Asia (e.g., Phau \& Min, 2009; Prendergast, Chuen, \& Phau, 2002). One region, however, was neglected - the Middle East. Little is known about how consumers in the region perceive counterfeits (versus original brands), their motivations to buy fake products or their relationship and experience with fake goods - aspects of a phenomenon which is strongly related to culture (Eisend \& Schuchert-Güler, 2006). This takes with surprise as countries in the region have a growing and young customer base. This makes them a particularly attractive target markets for branded products and at the same time easy prey to counterfeits (Ollier \& Behean, 2009).
\end{abstract}

In our paper, we try to shed light on the consumer perceptions of counterfeit products in a selected country (Iran) and a particular industry (sports gear). While past research on counterfeits has primarily focused on luxury brands (e.g., Han, Nunes, \& Drèze, 2010; Wilcox, Kim, \& Sen, 2009), where the symbolic value is predominant, sports gear represents a product category with considerable social risk (e.g., to be detected as someone who wears fakes; which were investigated in most previous studies) and functional risk at the same time (e.g., injuries through faulty fake products; malfunction due to bad quality) (Eisend \& Schuchert-Güler, 2006). Moreover, up to now, the specific retailing situation in the chosen country context makes it very hard to distinguish original from fake and thus facilitates deceptive counterfeiting.

As the geographic area and the specific product category investigated have not attracted attention so far, we decided to take a qualitative approach (qualitative interviews; $\mathrm{n}=15$ ). While no official figures exist, sales of counterfeits are rampant. Original sports gear manufacturers who sell their products in Iran try to protect their brand by selling through authorized channels only and relying on Iranian IPR law. We developed an interview guideline to focuse specifically on the role of brands versus counterfeits for the consumers, usage situations and the impact of the immediate social environment on their purchasing decision. Interviews were conducted in the local language by a bilingual research assistant and transcribed into English. Respondents were selected from various areas in Teheran based on age, gender and profession. Interviews were conducted either at home or at the work place in a comfortable atmosphere without any listeners.

As our results show, respondents see counterfeits as a regular product alternative to branded products and take a pragmatic approach to the product decision. Original and fake brands are often owned together, but then used at different occasions and circumstances. Consumers also developed certain heuristics (e.g., choice of shopping outlet, consultation with "expert" friends) to identify fakes. They choose fakes for pragmatic reasons (e.g., can be changed more often due to the lower price) or a lack of financial means. The social risk of being detected as someone who wears fakes does not seem to be a big issue for consumers, while functional risks such as health concerns are taken into consideration.

As to managerial implications, consumers cherish and value brands (particularly Adidas). So if they had money and would have the trust in the sales outlet they choose to buy an original not a fake, they would select the original. Therefore, selling original brands through authorized dealers AND keeping tight control of them would instill confidence in consumers. Health concerns were quite prominent in our interviews, offering another starting point for a communication campaign of brand manufacturers to counter fakes in these markets. While consumers cherish the German origin of Adidas, they at the same time realize that Adidas products are made in China etc. (as are the fake Adidas products). This undermines their confidence in the brand promise and may decrease their brand loyalty to the original.

References available upon request 